\title{
Exosomal miR-25-3p from mesenchymal stem cells alleviates myocardial infarction by targeting pro-apoptotic proteins and EZH2
}

\author{
Yi Peng ${ }^{1}$, Ji-Ling Zhao ${ }^{1}$, Zhi-Yong Peng ${ }^{1}$, Wei-Fang $X u^{1}$ and Guo-Long Yu (1)
}

\begin{abstract}
Mesenchymal stem cell (MSC) therapy is a promising approach against myocardial infarction (MI). Studies have demonstrated that MSCs can communicate with other cells by secreting exosomes. In the present study, we aimed to identify exosomal microRNAs that might contribute to MSC-mediated cardioprotective effects. Primary cardiomyocytes were deprived of oxygen and glucose to mimic $\mathrm{Ml}$ in vitro. For the animal model of $\mathrm{Ml}$, the left anterior descending artery was ligated for $1 \mathrm{~h}$, followed by reperfusion for $12 \mathrm{~h}$. MSC-derived exosomes were used to treat primary cardiomyocytes or mice. Cardioprotection-related microRNAs were determined, followed by target gene identification and functional studies with quantitative PCR, western blotting, MTT assay, flow cytometry assay, chromatin immunoprecipitation and dual-luciferase assay. We found that MSC co-culture reduced OGD-induced cardiomyocyte apoptosis and inflammatory responses. Cardioprotection was also observed upon treatment with MSCderived exosomes in vitro and in vivo. In line with this, exosome uptake led to a significant increase in miR-25-3p in cardiomyocytes. Depletion of miR-25-3p in MSCs abolished the protective effects of exosomes. Mechanistically, miR25-3p directly targeted the pro-apoptotic genes FASL and PTEN and reduced their protein levels. Moreover, miR-25-3p decreased the levels of $\mathrm{EZH} 2$ and $\mathrm{H} 3 \mathrm{~K} 27$ me3, leading to derepression of the cardioprotective gene eNOS as well as the anti-inflammatory gene SOCS3. Inhibition of EZH2 or overexpression of miR-25-3p in cardiomyocytes was sufficient to confer cardioprotective effects in vitro and in vivo. We concluded that exosomal miR-25-3p from MSCs alleviated MI by targeting pro-apoptotic proteins and EZH2.
\end{abstract}

\section{Introduction}

Myocardial infarction (MI) denotes the presence of myocardial injury due to myocardial ischaemia ${ }^{1}$. Despite the awareness of the high risk of death, the mortality rate of acute MI (AMI) is steadily increasing in China ${ }^{2}$. According to the Institute for Health Metrics and Evaluation, ischaemic heart disease is the second leading cause of death in China, with a 54\% increase in the mortality rate from 2007 to 2017 (www.healthdata.org/ china). Progress in therapeutic research is hence eagerly desirable. Mesenchymal stem cell (MSC) therapy for MI

\footnotetext{
Correspondence: Guo-Long Yu (gl_y18@aliyun.com)

'Department of Cardiology, Xiangya Hospital, Central South University, Changsha 410008 Hunan Province, P.R. China
}

Edited by $\mathrm{G}$. Melino has been evaluated intensively in basic science and clinical trials. Pathologically, MI is characterized by myocardial cell death ${ }^{1}$ and pro-inflammatory responses ${ }^{3,4}$, which further exacerbate cardiac injury. MSCs, on the other hand, have been demonstrated to exert anti-apoptotic, regenerative and anti-inflammatory effects in animal models of $\mathrm{MI}^{5-9}$. Furthermore, a recent meta-analysis with data from 14 randomized placebo-controlled trials suggested that MSC therapy is safe and beneficial in terms of the improved left ventricular ejection fraction and regenerative outcomes ${ }^{6}$. These animal and clinical studies indicate that MSC treatment represents a promising approach for MI therapy. Therefore, revealing the mechanism by which MSCs exert protective effects in MI is of great significance. 
MicroRNAs belong to a subclass of noncoding RNAs that post-transcriptionally regulate the expression of protein-coding genes. Dysregulated expression of microRNAs is frequently reported in cardiac tissues post $\mathrm{MI}^{10,11}$, and dysregulated microRNAs have been considered diagnostic biomarkers of $\mathrm{MI}^{12,13}$. In addition, the cardioprotective roles of microRNAs have drawn increasing interest ${ }^{14-16}$. For instance, microRNA-24 overexpression was demonstrated to counteract cardiomyocyte ${ }^{17}$ and endothelial cell ${ }^{10}$ apoptosis and initiate myocardial healing ${ }^{18}$ after MI. Likewise, microRNA-214 overexpression also appeared to be cardioprotective ${ }^{19,20}$. Nonetheless, delivery of microRNA to cardiac tissues is not an easy task, limiting the translation of microRNA from bench to clinic. In this regard, exosomes, which transfer cargos for cell-cell communication ${ }^{21-23}$, may be applied to efficiently deliver cargo exosomal microRNAs to target cells. Indeed, it was demonstrated that MSCderived exosomes contain more than 50 microRNAs, such as microRNA-1246 and microRNA-25-3p ${ }^{24}$. Therefore, whether MSCs mediate cardioprotection via exosomes and other exosomal microRNAs is of great interest. Indeed, great attention has been drawn to the finding that microRNAs appear to be indispensable for the therapeutic effects of MSC-derived exosomes in different disease models $^{24,25}$.

Enhancer of zest homologue 2 (EZH2) is a histonelysine $\mathrm{N}$-methyltransferase that is part of polycomb repressive complex 2 (PRC2). By the addition of methyl groups to histone $\mathrm{H} 3$ at lysine 27, EZH2 promotes the formation of heterochromatin and thereby represses gene expression $^{26}$. In a mouse model of limb ischaemia, EZH2 was upregulated accompanied by a reduction in eNOS and BDNF expression ${ }^{27}$. Inhibition of EZH2 by 3deazaneplanocin (DZNep) restored the levels of eNOS and BDNF, which then facilitated angiogenesis in ischaemic muscles. In addition, EZH2-mediated gene repression is involved in an exaggerated inflammatory response. It was reported that EZH2 deficiency promoted the expression of suppressor of cytokine signalling 3 (SOCS3) in macrophages and microglia ${ }^{28}$. SOCS3 in turn caused the degradation of Taf6, leading to suppression of nuclear factor $\kappa B(\mathrm{NF}-\kappa \mathrm{B})$ and its downstream proinflammatory responses. It should be noted that exaggerated inflammation is also evident after MI and deteriorates cardiac injury. EZH2 therefore might act as a critical modulator of MI by repressing eNOS and SOCS3.

In the current study, the roles of exosomes in MSCmediated cardioprotective effects were explored with in vitro and in vivo in models of MI. Our study demonstrated that MSC-derived exosomal miR-25-3p targeted pro-apoptotic genes to facilitate cardiomyocyte survival and targeted EZH2, thereby disinhibiting the expression of SOCS3, leading to suppression of inflammation post MI.

\section{Materials and methods \\ Culture of primary cardiomyocytes}

Primary cardiomyocytes were cultured and adopted for in vitro studies. All animal studies were approved by the animal ethics committees of Xiangya Hospital, Central South University (approval number: 201503435). Cardiomyocytes were isolated from adult $\mathrm{BALB} / \mathrm{c}$ mice (6-8 weeks old) according to a published protocol ${ }^{29}$. Heparin ( $5 \mathrm{IU} / \mathrm{g}$ body weight) was administered via intraperitoneal injection before heart isolation. The heart was then perfused with perfusion buffer (in $\mathrm{mM} ; \mathrm{NaCl}$, 120; $\mathrm{KCl}, 5.4 ; \mathrm{Na}_{2} \mathrm{HPO}_{4} .7 \mathrm{H}_{2} \mathrm{O}, 0.33 ; \mathrm{MgSO}_{4} .7 \mathrm{H}_{2} \mathrm{O}$, 246.48; taurine, 125.1; butanedione monoxide, 101.1; HEPES, 238.3; glucose, 180.16; EGTA, 0.4; insulin, $5 \times 10^{-5} \mathrm{U} / \mathrm{L}$ ) and digestion buffer (perfusion buffer without EGTA and with $\mathrm{CaCl}_{2}, 0.3 \mathrm{mM}$; protease XIV, $0.2 \mathrm{mg} / \mathrm{mL}$; collagenase II, $2.4 \mathrm{mg} / \mathrm{mL}$ ). The ventricles were isolated, minced with forceps and dissociated by pipetting. Then, the mixtures were filtered to obtain a single-cell suspension, followed by two rounds of gravity sedimentation to remove fibroblasts and endothelial cells in the supernatant. The purified cardiomyocytes were seeded in laminin $(10 \mu \mathrm{g} / \mathrm{mL})$-coated culture plates and maintained in Dulbecco's modified Eagle's medium (DMEM, Gibco, Grand Island, NY, US) supplemented with 10\% heatinactivated foetal bovine serum (FBS, Gibco, Grand Island, NY, US) and 1\% penicillin-streptomycin solution (Gibco, Grand Island, NY, US) in a humidified atmosphere of $5 \% \mathrm{CO}_{2}$ at $37^{\circ} \mathrm{C}$. Cells were tested without contamination with mycoplasma.

\section{Culture of primary MSCs}

MSCs were isolated from BALB/c mice (6-8 weeks old) according to a published protocol ${ }^{30}$. Bone marrow was flushed out and collected from the tibia and femur with a $10 \mathrm{~mL}$ syringe. The cell suspension was filtered through a 70-mm filter mesh and seeded in culture dishes at a density of $25 \times 10^{6}$ cells $/ \mathrm{mL}$ with complete DMEM. After $3 \mathrm{~h}$, the medium was replaced with fresh complete DMEM to remove haematopoietic cell lineages. Then, the medium was replaced with fresh complete DMEM every $8 \mathrm{~h}$ for up to $72 \mathrm{~h}$ of initial culture. When the remaining adherent cells reached $65-70 \%$ confluence ( $<2$ weeks), trypsin solution $(0.25 \%$, with $1 \mathrm{mM}$ EDTA) was added for $2 \mathrm{~min}$ at room temperature. The floating cells, i.e., MSCs, were collected for further culture. For MSC and cardiomyocyte co-culture experiments, cardiomyocytes were seeded on the bottom of each well, and MSCs were maintained in the cell culture inserts of the plate. Cells were tested without contamination with mycoplasma. 


\section{Transfection}

Short hairpin RNA (shRNA) targeting SOCS3 and its scramble control were designed by GenePharma (Shanghai, China). Oligos encoding the shRNA were then synthesized and inserted into the pGPH1 vector (GenePharma, Shanghai, China). MiR-25-3p mimics, inhibitors and their respective scramble control oligos were obtained from GenePharma (Shanghai, China). Cells were seeded onto 6-well plates. For transfection, the indicated plasmids and Lipofectamine $3000(3 \mu \mathrm{L} /$ well $)$ were mixed and added to cells. For miRNA studies, 25 pmol of microRNA mimics/inhibitor or their scramble controls (NC) was mixed with $7.5 \mu \mathrm{L}$ of Lipofectamine RNAiMAX reagent (Thermo Scientific, San Jose, CA, USA) and used for transfection. All the transfected cells were then cultured for $48 \mathrm{~h}$ before further assays unless indicated otherwise.

\section{Exosome isolation and characterization}

Exosomes were enriched from MSC culture media with Total Exosome Isolation Reagent (\#4478359, Thermo Scientific, San Jose, CA, USA). MSCs were cultured in DMEM containing 10\% exosome-depleted FBS (\#A2720803, Thermo Scientific, San Jose, CA, USA). The culture media were collected and centrifuged at $2000 \times g$ for $30 \mathrm{~min}$ to remove cells and debris. The supernatant was transferred to a new tube and mixed with isolation reagent $(\mathrm{v}: \mathrm{v}=2: 1)$ by vortexing thoroughly. The mixed solution was incubated at $4{ }^{\circ} \mathrm{C}$ overnight and then centrifuged at $10,000 \times g$ for 1 at $44^{\circ} \mathrm{C}$. The pellet consisting of total exosomes was resuspended in PBS and ready for use. For morphology visualization, freshly isolated exosomes were resuspended in $2 \%$ paraformaldehyde in cold PBS. Then, exosomes were mounted on copper grids, fixed with $1 \%$ glutaraldehyde in PBS, negatively stained with uranyl-oxalate solution ( $\mathrm{pH} 7$ ) for $5 \mathrm{~min}$, and embedded in methylcellulose solution. A transmission electron microscope was used to visualize exosomes. Size and size distribution profile of isolated exosomes was evaluated using a NanoSight NS500 instrument (NanoSight Technology, Malvern, UK). To further characterize the exosomes, western blotting was performed to detect the levels of exosome markers, i.e., HSP70, CD63 and CD9. Briefly, exosomes were lysed by RIPA buffer $(\mathrm{NaCl}, 150 \mathrm{mM}$; Triton $\mathrm{X}-100,1 \%$; sodium deoxycholate, 0.5\%; SDS, 0.1\%; Tris, $50 \mathrm{mM}, \mathrm{pH} 8.0$ ) supplemented with protease and phosphatase inhibitor cocktails (\#5872, Cell Signaling Technology, Danvers, MA, USA). The proteins were then resolved and visualized as described in the Western blotting section.

\section{Detection of exosomes uptake by cardiomyocytes}

Isolated exosomes were incubated with $3.3 \mu \mathrm{L}$ of Alexa FlourTM 488 C5 Maleimide $(200 \mu \mathrm{g} / \mathrm{mL}$, A10254, Thermo Scientific, San Jose, CA, USA) for $1 \mathrm{~h}$ at room temperature. The labelling was disturbed by passing through the exosome spin column (MW3000, 4484449, Thermo Scientific, San Jose, CA, USA), according to manufacturer's instruction. The labelled exosomes were washed out and resuspended with $1 \mathrm{~mL}$ of serum free OptiMEM (31985088, Thermo Scientific, San Jose, CA, USA). For each well in a 4-well plate, $250 \mu \mathrm{L}$ labelled exosomes were incubated with primary cardiomyocytes in the standard cell culture condition for $4 \mathrm{~h}$ at $37^{\circ} \mathrm{C}$. Cardiomyocytes were then counterstained with CellTracker Deep Red dye and mounted with ProLong Gold antifade mountants without DAPI (\#P36934, Thermo Scientific, San Jose, CA, USA). The cells labelling with Alexa 488 (green) under the microscope were considered as positive cells containing exosomes.

\section{In vitro oxygen-glucose deprivation (OGD) model}

Primary cardiomyocytes were cultured with glucosefree DMEM (\#11966025, Thermo Scientific, San Jose, CA, USA) in an anaerobic chamber $\left(1 \% \mathrm{O}_{2}, 5 \% \mathrm{CO}_{2}\right)$ at $37^{\circ} \mathrm{C}$ for the indicated hours to induce ischaemic injury. For exosome treatment, cells were treated with exosomes $6 \mathrm{~h}$ after OGD treatment at concentrations of $50 \mu \mathrm{g} / \mathrm{ml}$ exosomes.

\section{MTT assay}

Cardiomyocytes were seeded onto 96 -well plates at a density of $5 \times 10^{3}$ cells/well and treated as specified in the Results section. At the time point of the assay, $10 \mu \mathrm{L}$ of MTT solution (Sigma-Aldrich, St. Louis, MO, USA) in PBS $(5 \mathrm{mg} / \mathrm{mL})$ was added to each well and incubated in the cell culture incubator for $3 \mathrm{~h}$. The supernatant was removed carefully. The formazan crystals were then dissolved in $100 \mu \mathrm{L}$ of dimethyl sulfoxide (DMSO, Sigma-Aldrich, St. Louis, MO, USA). Cell viability in each well was determined by optical density measurement at $490 \mathrm{~nm}$.

\section{Annexin V/propidium iodide (PI) apoptosis assay}

Cardiomyocytes were seeded onto 12-well plates at a density of $1 \times 10^{5}$ cells/well. After treatment as specified in the Results section, the cells were trypsinized and harvested for staining using the Annexin V-FITC/PI Detection Kit, according to the manufacturer's instructions (Sigma-Aldrich, St. Louis, MO, USA). Cells were analysed by flow cytometry (Becton-Dickinson, Franklin Lakes, NJ, US). The FITC + /PI - fraction and FITC + /PI + fraction were considered apoptotic cells (early and late apoptosis, respectively).

\section{Western blotting assay}

Total protein was extracted with cell lysis buffer (50 mM Tris, $150 \mathrm{mM} \mathrm{NaCl}, 1 \% \mathrm{NP}-40,1 \mathrm{mM}$ EDTA, pH 7.6) containing a cocktail of protease and phosphatase 
inhibitors. The protein concentration was determined using a Pierce BCA protein assay kit (San Jose, CA, USA) according to the manufacturer's instructions. Samples ( $30 \mu \mathrm{g}$ protein/lane) were separated by SDS-PAGE and then transferred onto PVDF membranes $(0.22 \mu \mathrm{m}$ pore, Roche). After blocking with TBST buffer ( $20 \mathrm{mM}$ Tris, $137 \mathrm{mM} \mathrm{NaCl}, 0.1 \%$ Tween-20, $\mathrm{pH}$ 8.0) containing 5\% non-fat milk, the membranes were incubated with primary antibodies against FASL (\#AB16982), PTEN (\#9188), EZH2 (\#5246), H3K27me3 (\#9733), histone H3 (\#4499), eNOS (\#32027), SOCS3 (\#52113), p-p65 (\#3033), p65 (\#8242), p-IкB (\#2859), COX-2 (\#12282) and $\beta$-actin (\#3700) overnight at $4{ }^{\circ} \mathrm{C}$. Except for anti-FASL antibody, all the primary antibodies were ordered from Cell Signaling Technology (Danvers, MA, USA) and diluted 1:1000. Anti-FASL antibody was obtained from Millipore (Burlington, MA, USA). Then, the membranes were incubated with secondary antibody (1:3000, Cell Signaling Technology, Danvers, MA, USA) for $1 \mathrm{~h}$ at room temperature. The protein bands were visualized using Immobilon Western Chemiluminescent HRP substrate (Millipore, Burlington, MA, USA). The proteins were quantified using Quantity One software (Bio-Rad Laboratories, Inc., Hercules, CA, USA).

\section{RNA isolation and quantitative PCR (qPCR)}

Total RNA from cells was extracted with TRIzol reagent (Thermo Fisher Scientific, San Jose, CA, US) according to the manufacturer's instructions. Briefly, cells were resuspended in $1 \mathrm{~mL}$ TRIzol, followed by total RNA extraction with $200 \mu \mathrm{L}$ chloroform and RNA precipitation with $500 \mu \mathrm{L}$ isopropanol. Complementary DNA (cDNA) was synthesized from $1 \mu \mathrm{g}$ of total RNA using the PrimeScript RT reagent Kit (Takara, Dalian, CN). For microRNA extraction from exosomes, the miRNeasy Serum/Plasma Kit (QIAGEN, Germantown, MD, US) was used. QIAzolLysis Reagent $(1 \mathrm{~mL})$ was mixed with $200 \mu \mathrm{L}$ exosome to allow exosome lysis at room temperature for $5 \mathrm{~min}$. This was followed by $200 \mu \mathrm{L}$ chloroform treatment and microRNA enrichment with a RNeasy MinElute spin column. A TaqMan microRNA reverse transcription kit (Thermo Fisher Scientific, San Jose, CA, US) was then used to reverse transcribe miRNA to cDNA. cDNAs were diluted 20-fold with $\mathrm{ddH}_{2} \mathrm{O}$ and used for qPCR with a SYBR Premix EX Ta kit (Takara, Dalian, CN) in an ABI 7500HT real-time PCR system (Thermo Fisher Scientific, San Jose, CA, US). The gene expression levels in all samples were normalized to U6 snRNA (for miRNA) or $\beta$-actin (for mRNA) expression levels using the $2^{-\Delta \Delta C t}$ method. The primer sequences in the current study are listed in Table 1.

\section{Chromatin Immunoprecipitation (ChIP)-qPCR assay}

A ChIP-qPCR assay was performed to assess the binding of EZH2 or $\mathrm{H} 3 \mathrm{~K} 27 \mathrm{me} 3$ to the transcription regulatory
Table 1 Primers list.

\begin{tabular}{|c|c|c|}
\hline Gene & Forward primer & Reverse primer \\
\hline $\mid L-1 \beta$ & GCCACCTTTTGACAGTGATGAG & AAGGTCCACGGGAAAGACAC \\
\hline IL-6 & TGCAAGAGACTTCCATCCAG & TCCACGATTTCCCAGAGAAC \\
\hline TNF-a & CCGATGGGTTGTACCTTGTC & TGGAAGACTCCTCCCAGGTA \\
\hline eNOS & AGCATACCCCCACTTCTGTG & GAAGATATCTCGGGCAGCAG \\
\hline sOCS3 & GCTCCAAAAGCGAGTACCAG & TGACGCTCAACGTGAAGAAG \\
\hline $\begin{array}{l}\text { eNOS } \\
\text { (CHIP) }\end{array}$ & CCAGGAGTTCTTGTATGTATGG & GGTCCTTCTGTGATGTGGC \\
\hline $\begin{array}{l}\text { SOCS3 } \\
\text { (CHIP) }\end{array}$ & CGCTTCGGGACTAGGTAGGA & AGAAACCGGGAAAAGCTCCC \\
\hline
\end{tabular}

regions of the eNOS and SOCS3 genes. ChIP assays were conducted with ChIP-IT express kits (Active Motif, Shanghai, $\mathrm{CN}$ ) according to the manufacturer's instructions. Cardiomyocytes were seeded onto $10 \mathrm{~cm}$ dishes at a density of $1 \times 10^{7}$ cells/dish and treated as specified in the Results section. After treatment, cells were cross-linked with formaldehyde and lysed for chromatin isolation. The fixed chromatin was then sheared by enzymatic shearing cocktail (provided in the kit), incubated with anti-EZH2 or antiH3K27me3 and captured by protein G-conjugated magnetic beads. This was followed by chromatin elution, crosslinking reversal and proteinase $\mathrm{K}$ digestion. The products were then ready for qPCR using primers listed in Table 1.

\section{Dual-luciferase reporter assay}

Reporter assays were performed to investigate the regulation of the FASL, PTEN or EZH2 3'UTR by miR-25. Oligos containing the predicted miR-25 binding sites on the FASL, PTEN or EZH2 3'UTR and their corresponding mutant forms were cloned into the pmirGLO vector (Promega, Madison, WI, US). miR-25 mimics were applied for miR-25 overexpression. The pRL vector was used as the transfection control. During the transfection process, miR-25 mimics, pmirGLO vectors and pRL vector (25:25:1) were co-transfected into HEK293 cells with Lipofectamine 3000 at a ratio of 1:3 (DNA: Lipofectamine). As a control, miR-25 scramble oligos were also used for co-transfection in a separate experiments as described above. miR-25 mimics or scramble control were obtained from GenePharma (Shanghai, China). After transfection for $48 \mathrm{~h}$, firefly luciferase activity was determined and adjusted by Renilla luminescence using the assay kit according to the manufacturer's instructions (Promega, Madison, WI, US).

\section{Mouse model of ischaemia-reperfusion (I/R) injury}

All surgical procedures were approved by the animal ethics committees of Xiangya Hospital, Central South 
University (approval number: 201503435). $50 \mathrm{BALB} / \mathrm{c}$ mice (male, 20-22 g, 6-8 weeks) were randomly grouped (five groups, $n=10$ ) to establish $\mathrm{I} / \mathrm{R}$ injury models. The surgeries followed the procedures described previously (Zhaobin Xu, 2018). The investigator was blinded to the group allocation during the experiment. Briefly, thoracotomy was performed to expose the left anterior descending artery(LAD), which was tightly ligated together with PE-10 tubing for $1 \mathrm{~h}$ (ischaemia), and then released to allow reperfusion for $12 \mathrm{~h}$. Mice which did not recovered from I/R surgery were excluded. To explore the therapeutic effects of MSCderived exosomes, isolated exosomes ( $5 \mu \mathrm{g}$ in $100 \mu \mathrm{L}$ PBS) or PBS were injected into the border zone of the infarcted heart at three sites $30 \mathrm{~min}$ after ligation.

\section{Statistical analysis}

All experiments were performed at least three times. Data are presented as the mean \pm standard deviation (SD) based on three independent experiments. All statistical analyses were carried out using GraphPad Prism 6 (GraphPad Software, Inc., San Diego, CA, USA). All data were in a normal distribution, and variance was similar between the groups that are being statistically compared. Statistical evaluation was performed using Student's $t$ test (two-tailed) between two groups or one-way analysis of variance (ANOVA) followed by Tukey's post hoc test for multiple comparisons. $P<0.05$ was considered statistically significant in all cases.

\section{Results}

MSC co-culture reduced OGD-induced apoptosis and cytokine expression in cardiomyocytes

The MTT assay demonstrated that cardiomyocyte viability gradually decreased in a time-dependent manner after OGD (Fig. 1a), indicative of a successful OGD insult. OGD for $12 \mathrm{~h}$ was chosen for subsequent in vitro

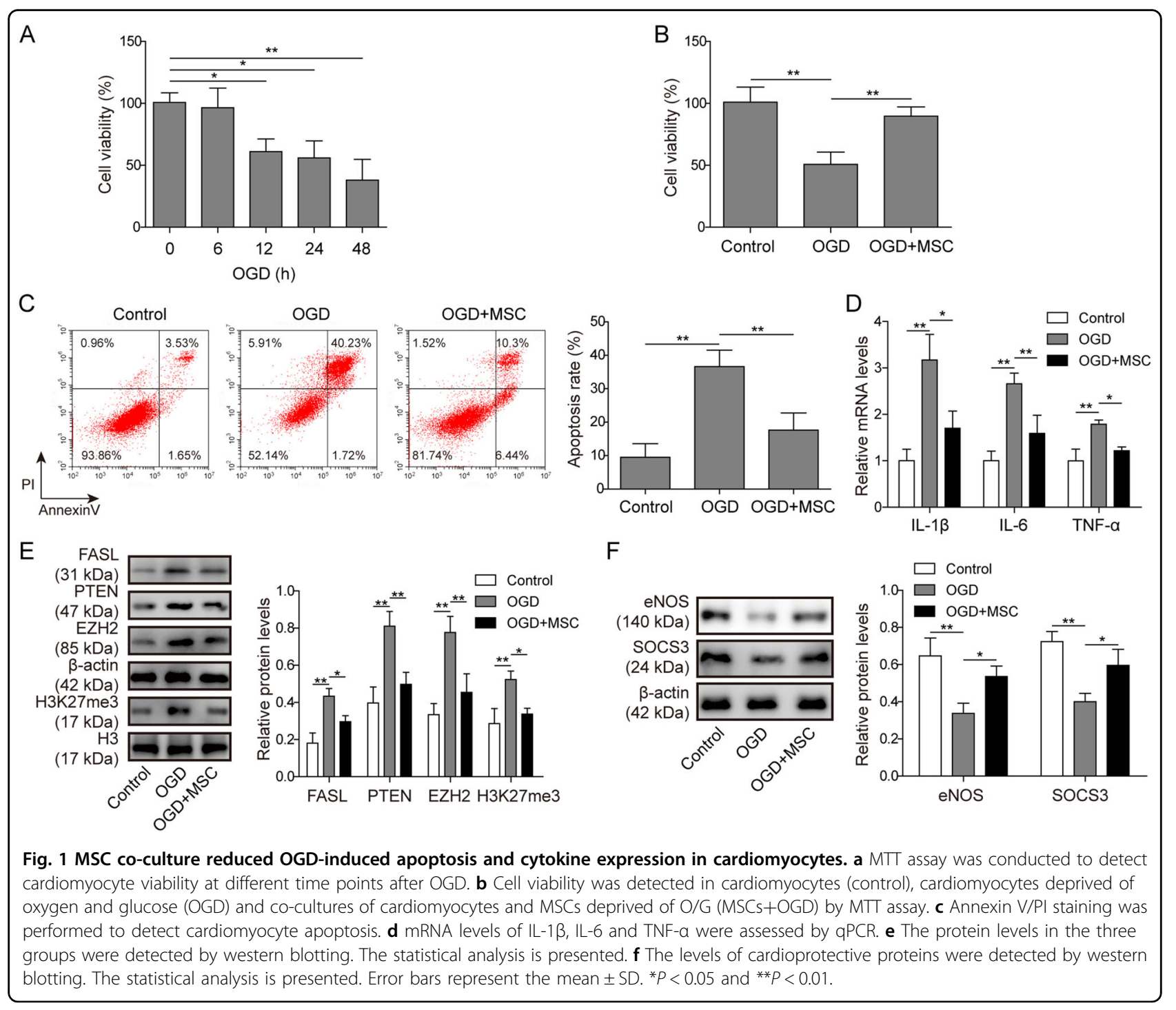


experiments. To explore the protective actions of MSCs, cardiomyocytes were cultured alone or co-cultured with MSCs. As expected, OGD significantly reduced cardiomyocyte viability compared with control (Fig. 1b). Coculture with MSCs, however, partially protected the cells from OGD-induced cell death. Similar findings were also obtained by the PI/Annexin V apoptotic assay. Compared with control, OGD caused dramatic accumulation of early and late apoptotic cardiomyocytes (Fig. 1c). The OGDinduced injury was significantly improved in the wells that were co-cultured with MSCs (Fig. 1c). Increase in proinflammatory cytokines is another characteristic of OGDinduced pathological alterations. qPCR demonstrated that OGD promoted the mRNA expression of IL- $1 \beta$, IL- 6 and TNF- $\alpha$ in cardiomyocytes compared with control cells (Fig. 1d). In the presence of MSCs, OGD-induced cytokine upregulation was greatly repressed (Fig. 1d). MSCmediated cardioprotection was also evaluated by detecting OGD-related protein expression. As expected, OGD led to significantly higher levels of pro-apoptotic proteins, i.e., FASL and PTEN in cardiomyocytes compared with normal culture conditions (Fig. 1e). In addition, the protein levels of EZH2, a component of $\mathrm{PRC} 2$, and its target H3K27me3 were also elevated by OGD (Fig. 1e). Consistent with the cell death assay, MSC co-culture significantly suppressed OGD-induced upregulation of all these pro-apoptotic proteins (Fig. 1e). In contrast to the effect of OGD on cell death-related proteins, OGD decreased the protein levels of eNOS and SOCS3 (Fig. 1f), which have cardioprotective effects. MSC co-culture partially recovered the levels of these pro-survival proteins in cardiomyocytes after OGD (Fig. 1f).

\section{Uptake of MSC-derived exosomes led to microRNA increase in cardiomyocytes}

Given that MSC-mediated cardioprotection is independent of direct cell-cell contact, we explored whether MSC-derived exosomes might play a role. Exosomes were isolated from the culture media of MSCs. TEM and western blotting confirmed that the purified product was highly enriched for exosomes (Fig. 2a, b). Size and size distribution showed a unimodal distribution of isolated particles with an average diameter $\sim 100 \mathrm{~nm}$, consistent with the definition of exosomes (Fig. 2c). Immunofluorescence staining showed that a strong Alexa 488 signal appeared in the cytoplasm of cardiomyocytes (Fig. 2d). As exosomes may act as sources of exogenous RNAs, the levels of miRNAs that were predicted to interact with EZH2 mRNAs (www.targetscan.org), were detected. qPCR showed that MSC-derived exosomes were enriched for a series of miRNAs, particularly miR-25-3p (Fig. 2e). The intracellular levels of miR-25-3p, miR-26b-3p and miR-137-3p were repressed in cardiomyocytes by OGD, while miR-144-3p was elevated, and miR-101-3p and
miR-138-3p did not change (Fig. 2e). Exosomal miRNAs, in particular, miR-25-3p, were dramatically elevated after exosome incubation (Fig. 2g). This result implied that the addition of MSC-derived exosomes might provide increased miR-25-3p to modulate the molecular network of cardiomyocytes.

\section{MSC-derived exosomes conferred cardioprotection by supplementing miR-25-3p}

To explore the roles of miR-25-3p in MSC-derived exosomes, a miRNA inhibitor was applied. As demonstrated in Fig. 3a, transfection with a miR-25-3p-specific inhibitor efficiently reduced the level of miR-25-3p in MSCs compared with the sham inhibitor or no treatment group. Notably, the exosomes isolated from MSCs transfected with miR-25-3p inhibitor also contained significantly lower levels of miR-25-3p than the exosomes from inhibitor NC-treated MSCs (Fig. 3a). Three groups of exosomes: those from MSCs without transfection (EXO), transfected with negative control inhibitor (EXO/ inhibitor NC) or transfected with miR-25-3p inhibitor (EXO/miR-25-3p inhibitor) were isolated for a series of functional assays. Compared with the OGD alone group, MSC-derived EXO significantly increased cardiomyocyte viability after OGD in the MTT assay (Fig. 3b). A similar effect was also observed in the cells treated with EXO/ inhibitor NC (Fig. 3b). However, cardioprotection was reversed when the cells were treated with an $\mathrm{EXO} / \mathrm{miR}$ 25-3p inhibitor. The apoptosis rates were also assessed. As shown in Fig. 3c, d, while EXO or EXO/inhibitor NC significantly reduced OGD-induced early and late apoptosis, comparable apoptotic rates were observed in the cardiomyocytes treated with OGD alone or OGD + EXO/ miR-25-3p. These differences between groups were also supported by the western blotting assay (Fig. 3g). OGD caused the accumulation of the pro-apoptotic proteins FASL and PTEN compared with the control treatment. Pre-treatment with EXO or EXO/inhibitor NC abolished this effect. However, depletion of miR-23-3p in exosomes failed to reduce the protein levels of FASL and PTEN compared with inhibitor NC group. These findings suggested that MSC-derived exosomes contributed to MSCmediated anti-apoptotic effects and that miR-25-3p played a critical role in these effects. The effects of exosomes on cytokine expression were detected by qPCR. As expected, both EXO and EXO/inhibitor NC significantly reversed OGD-induced IL-1 $\beta$, IL- 6 and TNF- $\alpha$ upregulation (Fig. 3e). However, the exosome-mediated antiinflammatory effects were abolished by pre-treatment with miR-25-3p inhibitor, as indicated by the comparison between the $\mathrm{EXO} /$ inhibitor $\mathrm{NC}$ and $\mathrm{EXO} / \mathrm{miR}-25-3 \mathrm{p}$ inhibitor groups (Fig. 3e). The NF- $\kappa$ B pathway, which is highly associated with cytokine expression, was hence investigated using a western blotting assay. OGD 
A

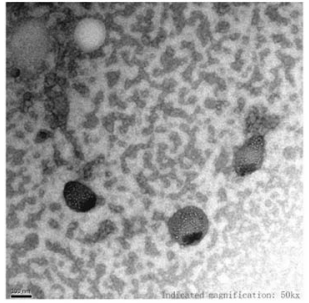

B

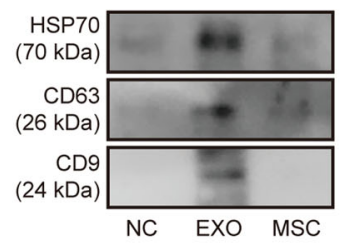

C

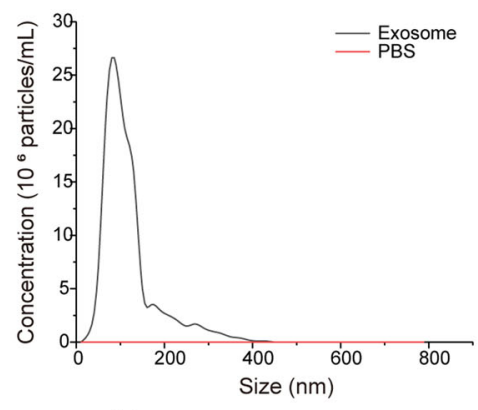

E
D
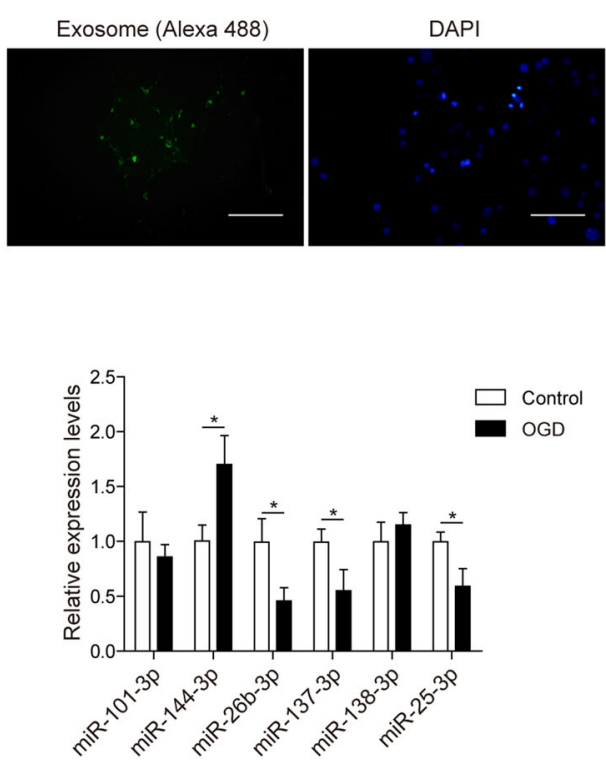

Merge

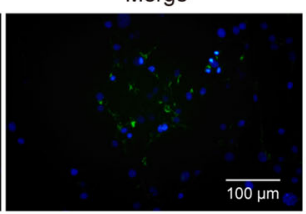

G
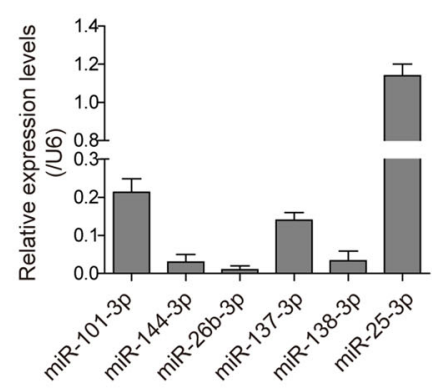

F

Fig. 2 Uptake of MSC-derived exosomes led to microRNA increase in cardiomyocytes. a Representative images of exosomes from MSCs. b
Exosomes were validated by assessing exosomal markers. c Size distribution of exosomes by nanosight. $\mathbf{d}$ Alexa 488 -labelled exosomes (green) were
observed in the cytoplasm of primary cardiomyocytes. e microRNA expression in isolated exosomes. $\mathbf{f}$ miR-25 in OGD-treated cardiomyocytes was
detected by $\mathrm{PPCR}$. $\mathbf{g}$ The levels of microRNAs in cardiomyocytes were detected by qPCR after exosome addition. Error bars represent the mean \pm SD.
${ }^{*} P<0.05$ and ${ }^{* *} P<0.01$. NC, negative control, denotes the exosome isolation reagent control; Exo, exosome. promoted phosphorylation of $\mathrm{p} 65$ (NF- $\mathrm{kB})$ and its upstream modulator $\mathrm{I} \kappa \mathrm{B}$, while the total protein of $\mathrm{p} 65$ and $I \kappa B$ remained unchanged (Fig. 3f). Pre-treatment with EXO or EXO/inhibitor $\mathrm{NC}$ significantly reduced the phosphorylation of p65 and IKB after OGD. However, the inhibition of the NF- $\mathrm{kB}$ pathway in the EXO/miR-25-3p inhibitor group was diminished compared with that in the EXO/inhibitor NC group (Fig. 3f).

\section{Exosomes disinhibited EZH2-mediated repression of cardioprotective genes by introducing miR-25-3p}

MSC co-culture may recover cardioprotective gene expression by inhibiting EZH2. We asked if miR-25-3p accounted for these effects. OGD increased the protein level of EZH2 and the trimethylation level at H3K27 (Fig. 4a). Consistent with the findings of MSC co-culture studies, EXO or EXO/inhibitor NC treatment was sufficient to suppress the OGD-induced increase in EZH2 and H3K27me3 (Fig. 4a). In contrast, depletion of miR-25-3p in the exosomes completely abolished this effect, as indicated by the comparison among OGD, OGD + EXO/ inhibitor NC and OGD + EXO/miR-25-3p inhibitor (Fig. 4a). The alterations of EZH2 were further validated by detecting the transcription of the target genes eNOS and SOCS3. OGD significantly reduced the mRNA (Fig. 4b) and protein (Fig. 4c) levels of these cardioprotective genes. In contrast, EXO and EXO/inhibitor NC, but not EXO/ miR-25-3p, recovered their expression (Fig. 4b, c). ChIP assays confirmed that both EZH2 and H3K27me3 were recruited to the promoter regions of eNOS and SOCS3 after OGD (Fig. 4d). Compared with that after OGD alone, recruitment of EZH2 and H3K27me3 to these promoter regions was significantly reduced after OGD + EXO or OGD + EXO/inhibitor NC treatment (Fig. 4d), 


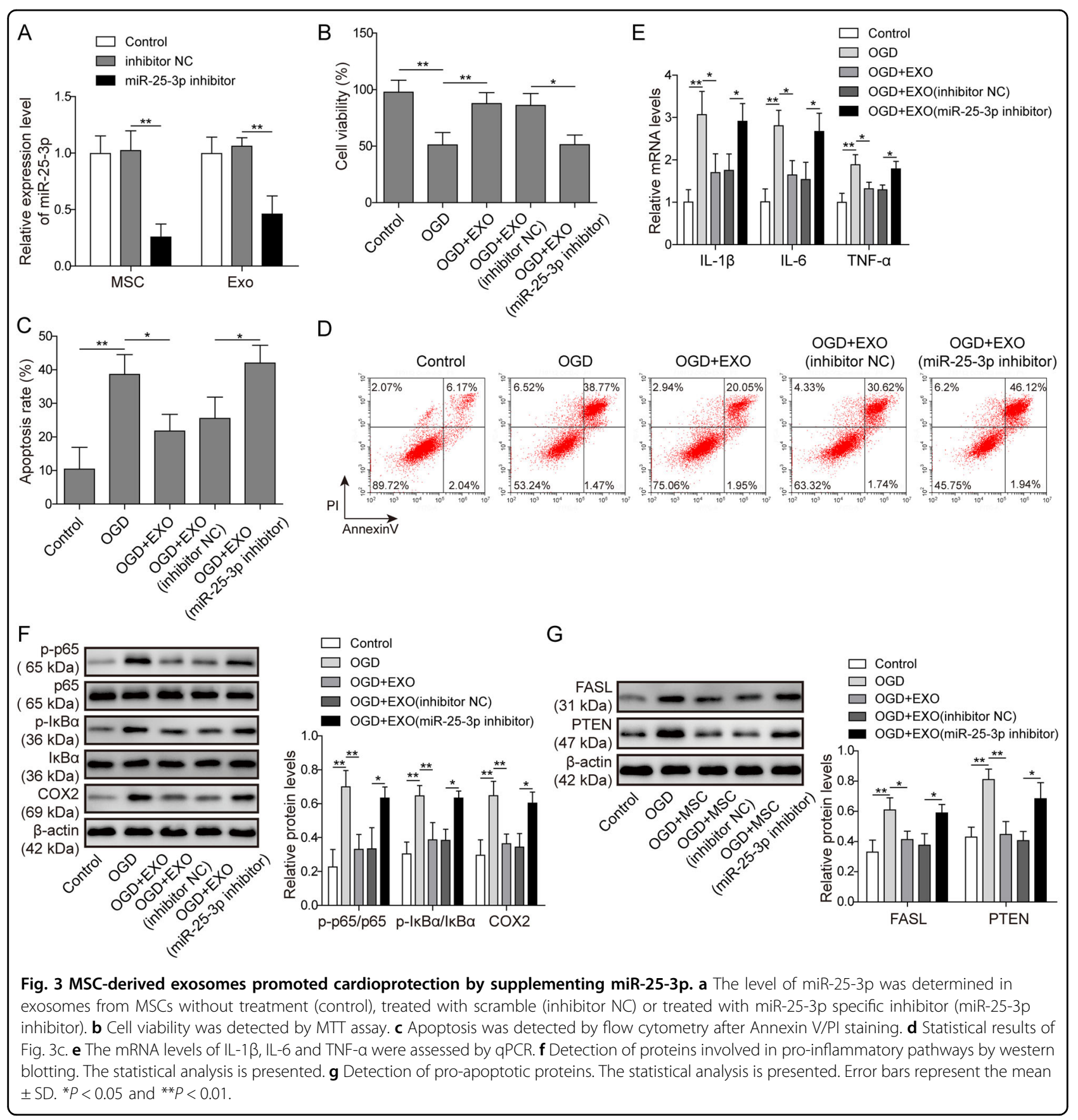

while exosomes without miR-25-3p failed to block the binding of EZH2 and H3K27me3 to these promoter regions (Fig. 4d).

miR-25-3p protected cardiomyocytes against OGDinduced apoptosis and increase in pro-inflammatory cytokines

To further validate the cardioprotective effects of exosomal miR-25-3p, forced expression of miR-25-3p was applied to explore its molecular functions. In the MTT assay (Fig. 5a), transfection of the miR-25-3p mimic (miR25 mimic) significantly impaired OGD-induced cytotoxicity compared with mimic NC transfection (Fig. 5a). In the $\mathrm{PI} /$ Annexin $\mathrm{V}$ assay, miR-25 mimic transfection significantly protected cardiomyocytes from OGD-induced apoptosis (OGD + mimic NC vs OGD + miR-25-3p mimic, Fig. 5b, c). Moreover, qPCR demonstrated that miR-25 mimic but not mimic NC blocked the upregulation of IL-1 $\beta$, IL- 6 and TNF- $\alpha$ after OGD (Fig. 5d), indicating that miR-25 exerted anti-inflammatory effects. The 


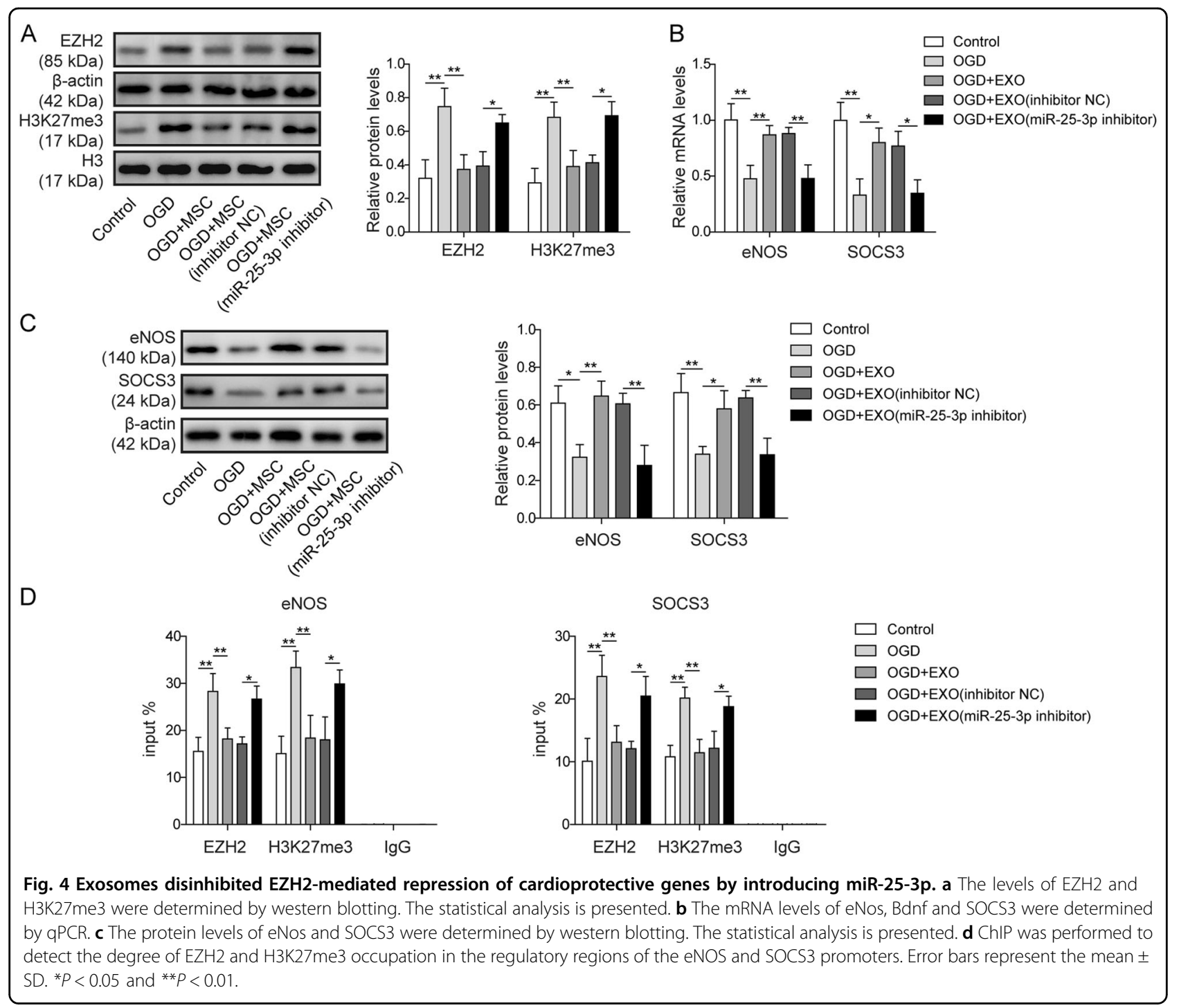

effects of miR-25-3p on apoptosis-related proteins were also assessed. Compared with mimic NC, miR-25-3p overexpression significantly attenuated OGD-induced upregulation of FASL, PTEN and EZH2 (Fig. 5e). In contrast, transfection of miR-25-3p inhibitor alone was sufficient to augment the mRNA levels of these proapoptotic modulators (Fig. S1). We then asked whether miR-25-3p might directly target the mRNA of these genes for degradation. Bioinformatic analysis revealed that the $3^{\prime}$ UTRs of all these genes harboured putative binding sites of the miR-25-3p seed sequence (Fig. 5f-h). The $3^{\prime} U T R$ fragments containing the putative binding sites (wild type, WT) or their mutant forms (Mut) were synthesized and cloned into the pmirGLO vector. Dual luciferase assays demonstrated that transfection of miR-25-3p mimic significantly reduced the luminescence of the three WT plasmids compared with mimic NC transfection (Fig. $5 f-h)$. The repression effects of the miR-25-3p mimic were abolished when the Mut plasmids were cotransfected (Fig. 5f-h). Notably, the addition of exosomes containing miR-25-3p was sufficient to reduce the luminescence of the three WT plasmids compared with the vehicle control (Fig. S2). These results confirmed that FASL, PTEN and EZH2 were direct targets of miR-25-3p.

Inhibition of EZH2 alleviated OGD-induced apoptosis and inflammation in cardiomyocytes at least partly through SOCS3

Given that EZH2 was shown to be a target of miR-253p, we explored the roles of EZH2 in OGD-induced cardiomyocyte injury. SOCS3 is one of the target genes of EZH2 and is frequently involved in cell survival and inflammatory pathways. Therefore, whether SOCS3 played a role underlying EZH2-mediated OGD events was also investigated. DZNep was used to inhibit EZH2 activity. To knock down SOCS3, shRNA selectively 


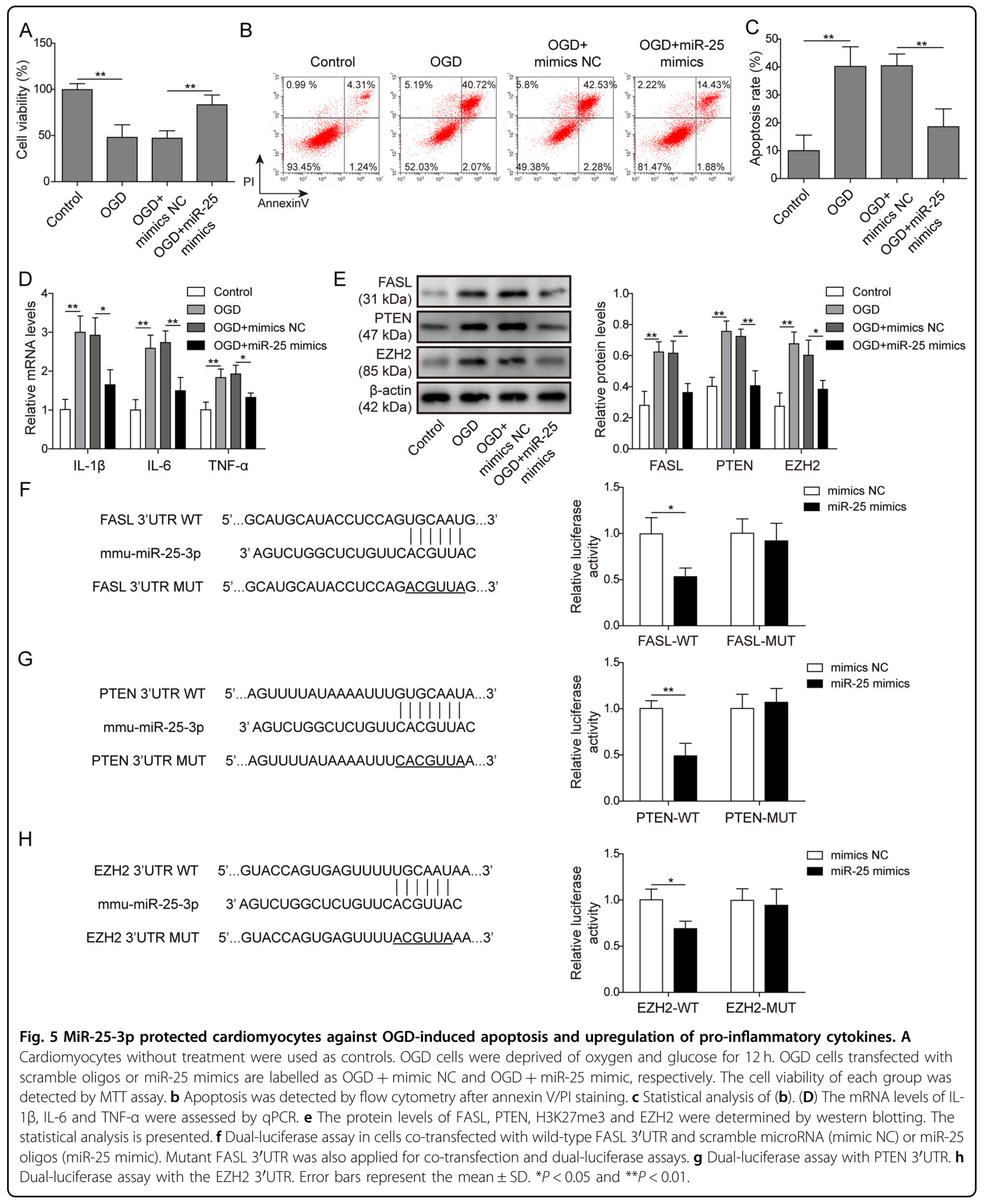

targeting SOCS3 (shSOCS3) or its scramble control (shNC) was transfected into cardiomyocytes prior to DZNep administration. MTT assays showed that EZH2 inhibition by DZNep partially rescued OGD-induced cell death (OGD vs OGD + DZNep, Fig. 6a). Transfection with shNC did not affect the cardioprotection-like effects 


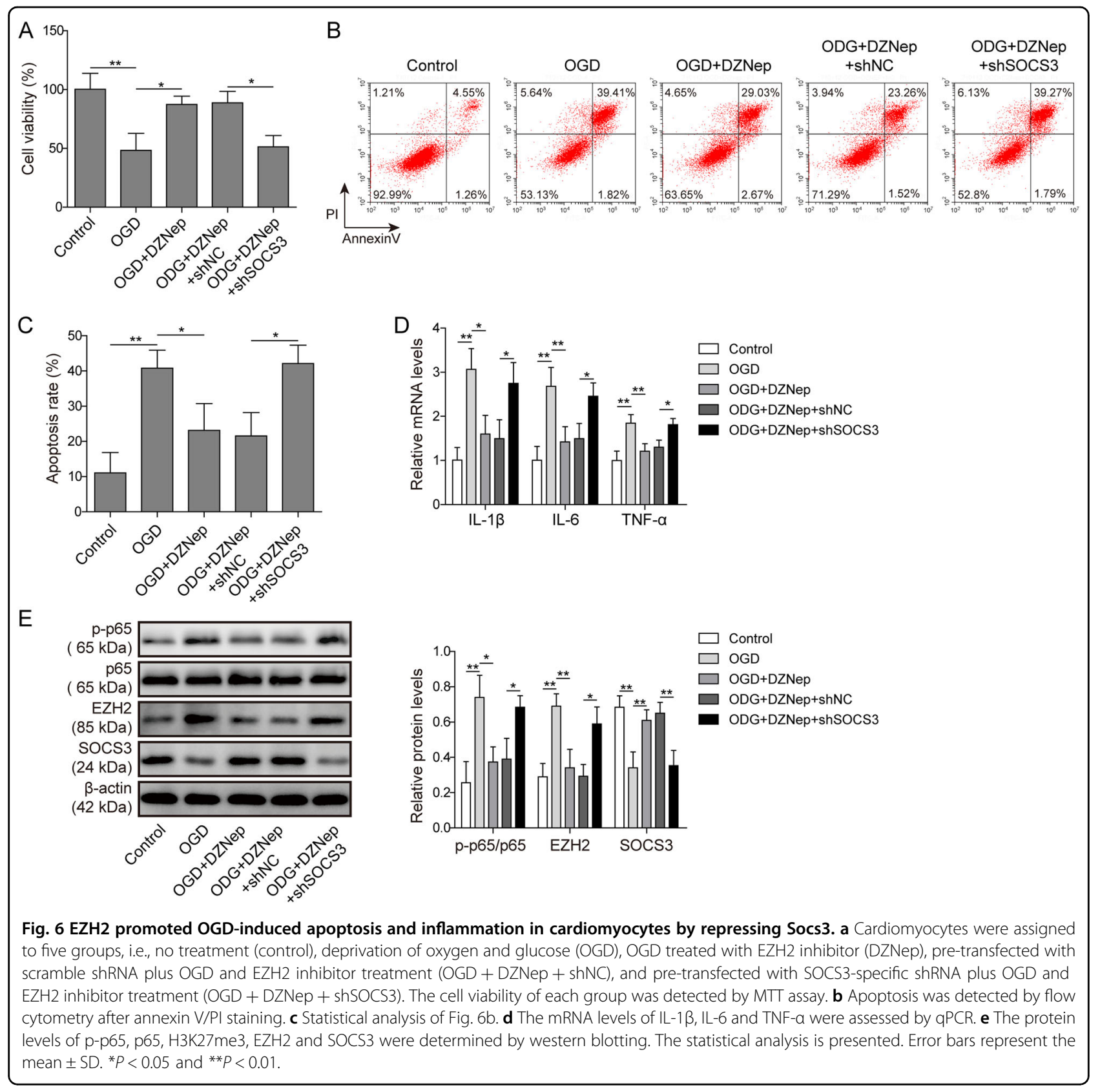

of DZNep (OGD + DZNep vs OGD + DZNep + shNC, Fig. 6a). Compared with the OGD + DZNep + shNC group, knockdown of SOCS3 abolished DZNep-mediated cardioprotection after OGD (OGD + DZNep + shNC vs OGD + DZNep + shSOCS3, Fig. 6a). This result implied that SOCS3 was at least partly required for the protective effect of EZH2 inhibition. Similar findings were obtained in the apoptosis assay (Fig. 6b, c). Compared with OGD alone, DZNep administration decreased the apoptotic rates after OGD. Knockdown of SOCS3 compromised the anti-apoptotic actions of EZH2 inhibition. Moreover, EZH2 inhibition by DZNep reversed OGD-induced IL-1 $\beta$,
IL- 6 and TNF- $\alpha$ upregulation. The anti-inflammatory actions were abolished by SOCS3 knockdown (Fig. 6d). Western blotting assays confirmed that DZNep efficiently reduced the upregulation of EZH2 and phosphorylation of p65 after OGD $(p<0.05$, OGD vs OGD + DZNep, Fig. $6 \mathrm{e})$, while knockdown of SOCS3 reversed these effects ( $p$ $<0.05$, OGD + DZNep + shNC vs OGD + DZNep + shSOCS3), indicative of the presence of feedback signalling. Our data suggested that inhibition of EZH2 alleviates OGD-induced apoptosis and inflammation, while knockdown of SOCS3 reversed this protective effect of the EZH2 inhibitor. 
MSC-derived exosomes elicited cardioprotection via miR25 in a mouse model of I/R injury

The therapeutic effects of MSC-derived exosomes were explored in a mouse model of I/R injury. TTC staining demonstrated that $I / R$ surgery induced substantial infarction in heart tissues (sham surgery vs $\mathrm{I} / \mathrm{R}$ ). Pretreatment with EXO or EXO/inhibitor NC significantly reduced the infarct area compared with I/R alone (Fig. 7a). However, the therapeutic effect of exosomes was abolished when miR-25-3p was depleted in exosomes $(\mathrm{I} / \mathrm{R}+$ $\mathrm{EXO} / \mathrm{miR}-25$ inhibitor vs $\mathrm{I} / \mathrm{R}+\mathrm{EXO} /$ inhibitor NC Fig. 7a). The expression of cytokines in heart tissues was also assessed. Consistent with the in vitro experiments, EXO or $\mathrm{EXO} /$ inhibitor $\mathrm{NC}$ efficiently reduced the upregulation of IL-1 $1 \beta$, IL-6 and TNF- $\alpha$ after I/R (Fig. 7b). The antiinflammatory actions of exosomes were decreased by miR-25 inhibition in exosomes (Fig. 7b). Finally, apoptosis- and inflammation-related proteins were detected in heart tissues from each group (Fig. 7c). I/R caused the accumulation of FASL, PTEN, EZH2 and H3K27me3 in injured tissues, whereas the SOCS3 level was repressed. Pre-administration of EXO or EXO/inhibitor, but not $\mathrm{EXO} / \mathrm{miR}-25-3 \mathrm{p}$ inhibitor, reversed the dysregulation of these proteins, which is consistent with the findings in the in vitro assay.

\section{Discussion}

The present study demonstrated that MSC-derived exosomes accounted for a vital part of the cardioprotective effects of MSCs by transferring miR-25-3p (Fig. 7d). By targeting FASL and PTEN, miR-25-3p reduced OGDinduced cardiomyocyte apoptosis. Moreover, miR-25-3p reversed ischaemic injury-induced EZH2 upregulation and consequently disinhibited the expression of SOCS3, leading to cardiomyocyte survival and inflammation suppression in vitro and in vivo (Fig. 7d).

Previous studies reported that the expression levels of miR-25 were associated with different subtypes of cardiovascular diseases ${ }^{31}$. An in vitro functional study showed that miR-25 overexpression was sufficient to protect against oxidative stress-induced cardiomyocyte death by inhibiting mitochondrial calcium upregulation ${ }^{32}$. In a murine model of sepsis, which induced substantial injuries to cardiac tissues, miR-25 overexpression targeted PTEN mRNA and promoted cardioprotection by counteracting apoptosis and inflammation ${ }^{33}$. Similar findings were obtained in the present study. MiR-25-3p overexpression by microRNA mimic transfection or exosome treatment not only facilitated cardiomyocyte survival but also suppressed detrimental inflammatory responses. In addition to PTEN inhibition, FASL suppression might also contribute to the cardioprotective effects of miR-25$3 p$. As these are tumour suppressor genes, it is not surprising that direct inhibition of the pro-apoptotic proteins
PTEN and FASL by miR-25-3p might reduce OGDinduced cardiomyocyte apoptosis.

In addition to its anti-apoptotic action, miR-25-3p upregulation through exosome incubation led to attenuated inflammation in the in vitro and in vivo models of MI. In the present study, we provided compelling evidence that the MI therapeutic actions of miR-25-3p were achieved by directly targeting EZH2, which was a novel finding in the current study. At least two mechanisms underlying miR-25-p/EZH2 axis-mediated effects were identified. As eNOS ${ }^{34-37}$ confers significant cardioprotection, the finding that inhibition of EZH2 by miR-25-3p reduced methylation at $\mathrm{H} 3 \mathrm{~K} 27$ and restored the expression of eNOS represents the first molecular mechanism. Our study demonstrated a coherent mechanism by which EZH2 overactivation deteriorates ischaemia-induced tissue injury by repressing eNOS and BDNF expression in a model of limb ischaemia ${ }^{27}$. Numerous studies have investigated the protective roles of eNOS in different models of ischaemia. The current study focused on SOCS3 instead.

The disinhibition of SOCS3 expression could be the second mechanism. Recently, it was revealed that EZH2, through H3K27 methylation, suppressed the expression of SERPINB1, which in turn promoted inflammationmediated prostate cancer progression ${ }^{38}$. This study highlighted a pro-inflammatory role of EZH2. In the current study, we reported that SOCS3 could be a target of EZH2 for inflammatory modulation. After miR-25-3p administration, EZH 2 and H3K27me3 occupation at the regulatory regions of the SOCS3 promoter was significantly reduced, indicative of SOCS3 restoration. SOCS3 in turn suppressed the expression of proinflammatory cytokines, which may aggravate cardiac injury. These findings were consistent with previous reports that SOCS3 plays a critical role in the antiinflammatory pathway in different scenarios of ischae$\mathrm{mia}^{39,40}$. Our study therefore indicated that the miR-25/ EZH2/SOCS3 axis was a critical inflammation modulatory network of MI and that EZH2 was the upstream regulator of SOCS3 acting as a potential target for MI therapy. It should be noted that several papers have reported a deleterious role of SOCS3 in a conditional knockout mouse model ${ }^{41,42}$. In these studies, the molecular function of SOCS3 has been closely linked to apoptosis in two mouse models of MI. Our study, however, revealed that SOCS3 played a pivotal role in exogenous miR-25 transfer (via MSC-derived exosomes) or EZH2 inhibitor-mediated suppression of inflammation, which is a crucial component of $I / R$ aetiology. The antiinflammatory roles of SOCS3 are consistent with most previous studies ${ }^{43,44}$. With respect to apoptosis, it is noteworthy that both miR-25 and EZH2 inhibitor reduced a series of pro-apoptotic genes, including Fas and PTEN, 
A

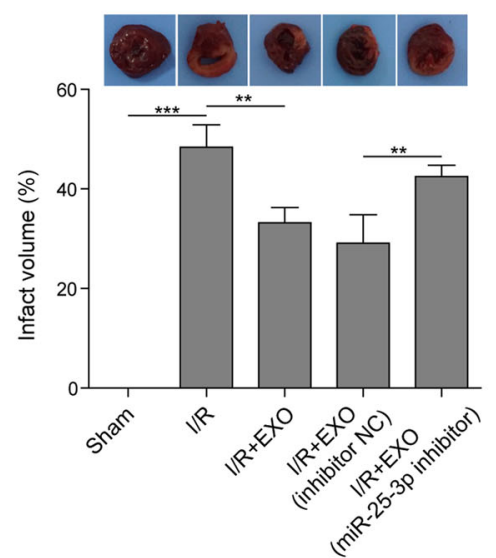

B

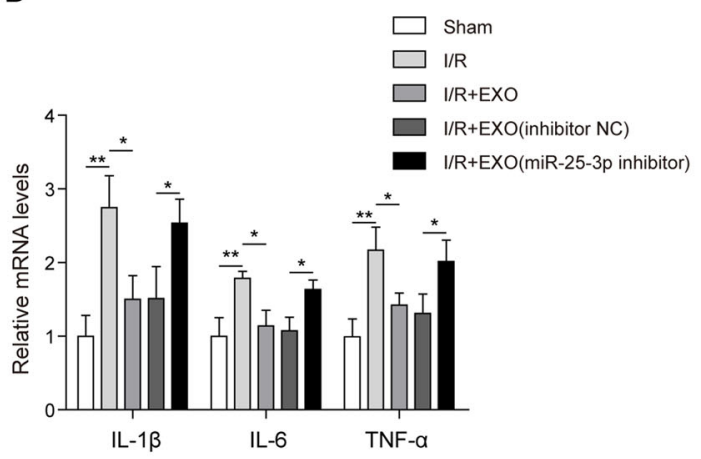

C
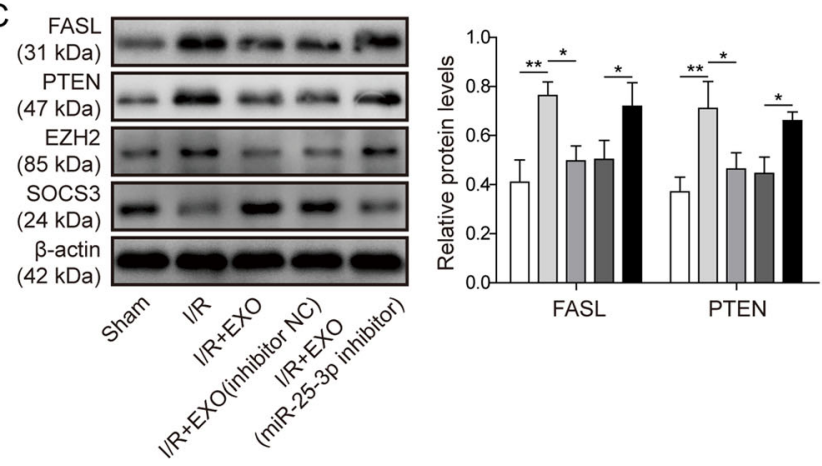

FASL

PTEN

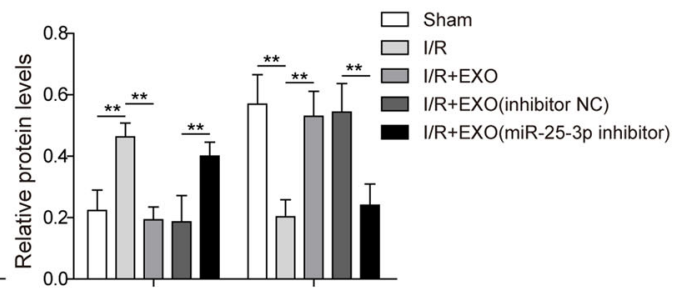

$\mathrm{EZH} 2$

socs3

D

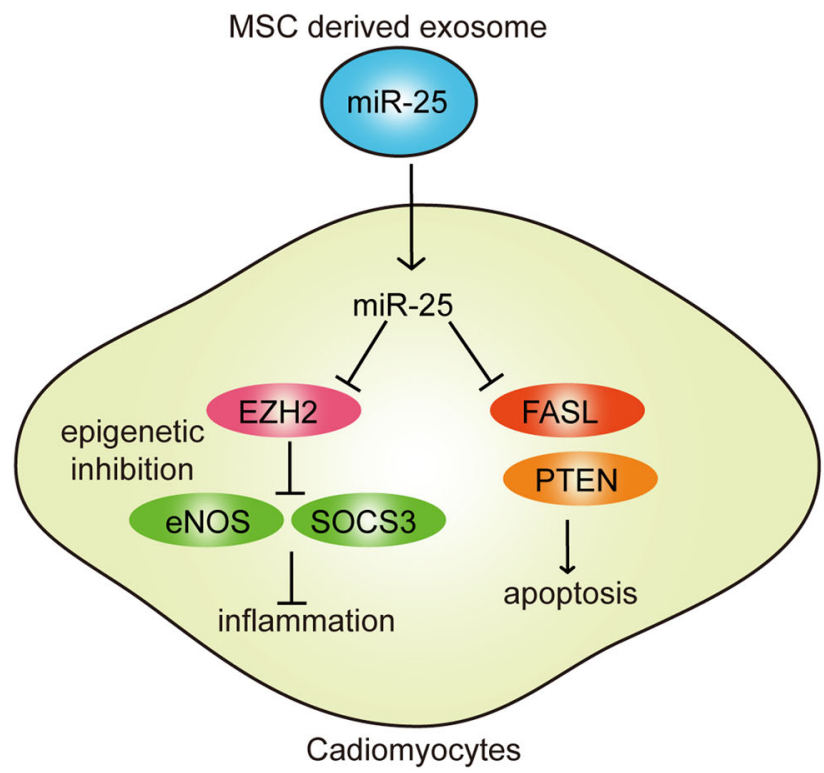

Fig. 7 MSC-derived exosomes exhibited cardioprotection via miR-25 in a mouse model of I/R injury. a Mice were randomly assigned to five groups, i.e., no treatment (sham), ischaemia injury alone (I/R), I/R treated with MSC-derived exosomes (I/R + EXO), I/R injected with exosomes from scramble oligo-treated MSCs (I/R + EXO/inhibitor NC) and I/R injected with exosomes from miR-25 inhibitor-treated MSCs (I/R + EXO/miR-25 inhibitor). Representative images of tissues stained with $\Pi T C$. The infarct areas from each group were compared. $\mathbf{b}$ The mRNA levels of IL-1 1 , IL-6 and TNF-a were assessed by qPCR. $\mathbf{c}$ The protein levels of FASL, PTEN, H3K27me3, EZH2 and SOCS3 were determined by western blotting. $\mathbf{d}$ A schematic representation of the current study. The statistical analysis is presented. Error bars represent the mean \pm SD. ${ }^{*} P<0.05$ and ${ }^{* *} P<0.01$. 
by reshaping the OGD-induced epigenome. Such alterations may counteract pro-apoptotic signalling in our model. Moreover, upregulation of SOCS3 has been considered to benefit animals or cells by suppressing inflammation and hence conferring anti-apoptotic effects in other ischaemia models ${ }^{45,46}$. We concluded that SOCS3 may play different roles under specific circumstances (i.e., initiation of I/R injury vs. therapy) or in specific components of aetiology (i.e., apoptosis vs. inflammation). In addition, contradictory roles for EZH2 in inflammation have also been reported. For instance, degradation of EZH2 by E3 ligase was demonstrated to facilitate recruitment of pro-inflammatory macrophages into colon tissues ${ }^{47}$. These studies implied that EZH2 exhibited distinct functionalities in different contexts of pathological alterations. More detailed studies are required prior to attempts to target EZH2-related signalling networks for the treatment of different diseases.

In the current study, we first demonstrated that MSCs were sufficient to reduce OGD-induced cell apoptosis and pro-inflammatory responses. Next, we found that exosomes secreted by MSCs accounted for a vital part of the cardioprotective effects of MSCs in vitro and in vivo. Moreover, we revealed that one of the exosomal cargoes, miR-25-3p, drove these effects of MSCs. Depletion of miR-25-3p in exosomes abolished the biological actions of these exosomes in vitro and in vivo. Hence, we propose that delivery of miR-25-3p to cardiomyocytes via exosomes represents another mechanism underlying MSCmediated cardioprotection. Furthermore, our study suggested that the preparation of miR-25-3p-containing exosomes could be a novel approach for the treatment of MI.

\section{Acknowledgements}

This work was supported by the Natural Science Foundation of China (No. 81570266)

\section{Conflict of interest}

The authors declare that they have no conflict of interest.

\section{Publisher's note}

Springer Nature remains neutral with regard to jurisdictional claims in published maps and institutional affiliations.

Supplementary Information accompanies this paper at (https://doi.org/ 10.1038/s41419-020-2545-6).

Received: 29 September 2019 Revised: 21 April 2020 Accepted: 22 April 2020

Published online: 05 May 2020

\footnotetext{
References

1. Thygesen, K. et al. Fourth universal definition of myocardial infarction (2018). Eur. Heart J. 40, 237-269 (2019).
}

2. Chen, W.-W. et al. China cardiovascular diseases report 2015: a summary. J. Geriatr. Cardiol. 14, 1-10 (2017).

3. Fang, L., Moore, X.-L., Dart, A. M. \& Wang, L.-M. Systemic inflammatory response following acute myocardial infarction. J. Geriatr. Cardiol. 12, 305-12 (2015).

4. Ong, S.-B. et al. Inflammation following acute myocardial infarction: multiple players, dynamic roles, and novel therapeutic opportunities. Pharmacol. Ther. 186, 73-87 (2018).

5. Huang, F. et al. Overexpression of microRNA-1 improves the efficacy of mesenchymal stem cell transplantation after myocardial infarction. Cardiology 125, 18-30 (2013).

6. El-Sayed Shafei, A. et al. Mesenchymal stem cell therapy: A promising cellbased therapy for treatment of myocardial infarction. https://doi.org/10.1002/ jgm.2995 (2017).

7. Miao, C., Lei, M., Hu, W., Han, S. \& Wang, Q. A brief review: the therapeutic potential of bone marrow mesenchymal stem cells in myocardial infarction. Stem Cell Res. Ther. 8, 242 (2017).

8. Peng, Y. et al. Effect of intravenous transplantation of hUCB-MSCs on M1/ M2 subtype conversion in monocyte/macrophages of AMI mice. Biomed. Pharmacother. 111, 624-630 (2019).

9. Jeong, $H$. et al. Mesenchymal stem cell therapy for ischemic heart disease: systematic review and meta-analysis. Int. J. Stem Cells 11, 1-12 (2018).

10. Fiedler, J. et al. MicroRNA-24 regulates vascularity after myocardial infarction. Circulation 124, 720-730 (2011).

11. Sun, T. et al. The role of microRNAs in myocardial infarction: from molecular mechanism to clinical application. Int. J. Mol. Sci. 18, 745 (2017).

12. D'Alessandra, Y. et al. Circulating microRNAs are new and sensitive biomarkers of myocardial infarction. Eur. Heart J. 31, 2765-2773 (2010).

13. Cheng, C., Wang, Q., You, W., Chen, M. \& Xia, J. MiRNAs as biomarkers of myocardial infarction: a meta-analysis. PLoS ONE 9, e88566 (2014).

14. Zhu, L.-P. et al. Hypoxia-elicited mesenchymal stem cell-derived exosomes facilitates cardiac repair through miR-125b-mediated prevention of cell death in myocardial infarction. Theranostics 8, 6163-6177 (2018).

15. Li, C.-C. et al. Endogenous reduction of miR-185 accelerates cardiac function recovery in mice following myocardial infarction via targeting of cathepsin K. J. Cell. Mol. Med. 23, 1164-1173 (2019).

16. $\mathrm{Hu}$, J. et al. MicroRNA-155 inhibition attenuates endoplasmic reticulum stressinduced cardiomyocyte apoptosis following myocardial infarction via reducing macrophage inflammation. Eur. J. Pharmacol. 857, 172449 (2019).

17. Qian, L. et al. miR-24 inhibits apoptosis and represses Bim in mouse cardiomyocytes. J. Exp. Med. 208, 549-60 (2011).

18. Wang, J. et al. MicroRNA-24 regulates cardiac fibrosis after myocardial infarction. J. Cell. Mol. Med. 16, 2150-2160 (2012).

19. Aurora, A. B. et al. MicroRNA-214 protects the mouse heart from ischemic injury by controlling $\mathrm{Ca}^{2+}$ overload and cell death. J. Clin. Invest. 122, 1222-32 (2012).

20. van Mil, A. et al. MicroRNA-214 inhibits angiogenesis by targeting Quaking and reducing angiogenic growth factor release. Cardiovasc. Res. 93, 655-665 (2012).

21. Bang, C. \& Thum, T. Exosomes: New players in cell-cell communication. Int. J. Biochem. Cell Biol. 44, 2060-2064 (2012).

22. Meldolesi, J. Current biology review exosomes and ectosomes in intercellular communication. https://doi.org/10.1016/j.cub.2018.01.059 (2018).

23. Mathieu, M., Martin-Jaular, L., Lavieu, G. \& Théry, C. Specificities of secretion and uptake of exosomes and other extracellular vesicles for cell-to-cell communication. Nat. Cell Biol. 21, 9-17 (2019).

24. Ferguson, $\mathrm{S}$. W. et al. The microRNA regulatory landscape of MSC-derived exosomes: a systems view. Sci. Rep. 8, 1419 (2018).

25. Reza-Zaldivar, E. E. et al. Potential effects of MSC-derived exosomes in neuroplasticity in Alzheimer's disease. Front. Cell. Neurosci. 12, 317 (2018).

26. Viré, E. et al. The Polycomb group protein EZH2 directly controls DNA methylation. Nature 439, 871-874 (2006).

27. Mitić, T. et al. EZH2 modulates angiogenesis in vitro and in a mouse model of limb ischemia. Mol. Ther. 23, 32-42 (2015).

28. Zhang, $X$. et al. Macrophage/microglial Ezh2 facilitates autoimmune inflammation through inhibition of Socs3. J. Exp. Med. 215, 1365-1382 (2018).

29. Louch, W. E., Sheehan, K. A. \& Wolska, B. M. Methods in cardiomyocyte isolation, culture, and gene transfer. J. Mol. Cell. Cardiol. 51, 288-98 (2011).

30. Soleimani, M. \& Nadri, S. A protocol for isolation and culture of mesenchymal stem cells from mouse bone marrow. Nat. Protoc. 4, 102-106 (2009). 
31. Sárközy, M., Kahán, Z. \& Csont, T. A myriad of roles of miR-25 in health and disease. Oncotarget 9, 21580-21612 (2018).

32. Pan, L. et al. MiR-25 protects cardiomyocytes against oxidative damage by targeting the mitochondrial calcium uniporter. Int. J. Mol. Sci. 16, 5420 (2015)

33. Yao, Y., Sun, F. \& Lei, M. miR-25 inhibits sepsis-induced cardiomyocyte apoptosis by targetting PTEN. Biosci. Rep. 38, BSR20171511 (2018)

34. Jugdutt, B. I. Nitric oxide and cardioprotection during ischemia-reperfusion. Heart Fail. Rev. 7, 391-405 (2002).

35. Farah, C. et al. Exercise-induced cardioprotection: a role for eNOS uncoupling and NO metabolites. Basic Res. Cardiol. 108, 389 (2013).

36. Farah, $\mathrm{C}$. et al. Key role of endothelium in the eNOS-dependent cardioprotection with exercise training. J. Mol. Cell. Cardiol. 102, 26-30 (2017).

37. Shinmura, K. et al. Indispensable role of endothelial nitric oxide synthase in caloric restriction-induced cardioprotection against ischemia-reperfusion injury. Am. J. Physiol. Circ. Physiol. 308, H894-H903 (2015).

38. Lerman, I. et al. Epigenetic suppression of SERPINB1 promotes inflammationmediated prostate cancer progression. Mol. Cancer Res. 17, 845-859 (2019).

39. Collino, $M$. et al. Peroxisome proliferator-activated receptor $\beta / \delta$ agonism protects the kidney against ischemia/reperfusion injury in diabetic rats. Free Radic. Biol. Med. 50, 345-353 (2011).
40. Wang, $X$. et al. miRNA-3473b contributes to neuroinflammation following cerebral ischemia. Cell Death Dis. 9, 11 (2018).

41. Oba, T. et al. Cardiac-specific deletion of SOCS-3 prevents development of left ventricular remodeling after acute myocardial infarction. J. Am. Coll. Cardiol. 59, 838-852 (2012)

42. Nagata, T. et al. Cardiac-specific SOCS3 deletion prevents in vivo myocardial ischemia reperfusion injury through sustained activation of cardioprotective signaling molecules. PLOS ONE 10, e0127942 (2015).

43. Jo, D., Liu, D., Yao, S., Collins, R. D. \& Hawiger, J. Intracellular protein therapy with SOCS3 inhibits inflammation and apoptosis. Nat. Med. 11, 892-898 (2005).

44. Carow, B. \& Rottenberg, M. E. SOCS3, a major regulator of infection and inflammation. Front. Immunol. 5, 58 (2014).

45. Wang, $\mathrm{X}$. et al. MiRNA-3473b contributes to neuroinflammation following cerebral ischemia article. Cell Death Dis. 9, 11 (2018).

46. Zhou, B., Liu, H. Y. \& Zhu, B. L. Protective role of SOCS3 modified bone marrow mesenchymal stem cells in hypoxia-induced injury of PC12 cells. J. Mol. Neurosci. 67, 400-410 (2019).

47. He, J. et al. Fbxw7 increases CCL2/7 in CX3CR1hi macrophages to promote intestinal inflammation. J. Clin. Invest. 130, 3877-3893 (2019). 